\title{
Dramatische Dispositive - vor der Zeit des Theaters?
}

\author{
Christiane Ackermann • Hartmut Bleumer
}

Online publiziert: 14. Oktober 2020

(C) Der/die Autor(en) 2020

Die Idee zu diesem Heft entstand im Zuge einer Diskussion über ein elementares epistemisches Problem der Ästhetik von Drama und Theater: Wie lassen sich diese als literatur- und kulturhistorische Gegenstände fassen, wenn schon deren Bezeichnung ein modernes Dispositiv aufruft und insofern die Sicht auf die vormodernen Phänomene präformiert - wenn sie es nicht historisch zu verzeichnen oder sogar zu verstellen droht? Auf den daraus hervorgegangenen Vorschlag, an einer auffälligen terminologischen Lücke im Bereich der Literatur- und Kulturwissenschaft zur Vormoderne zu arbeiten, haben sich die Beiträgerinnen und Beiträger des vorliegenden Heftes eingelassen. Diese Anregung - in einem ersten Essay zur Vorbereitung auf diese Ausgabe der LiLi formuliert - machte darauf aufmerksam, dass die medienund diskurstheoretische Karriere des Dispositiv-Begriffs gerade dort kaum Resonanz gefunden hat, wo sie historisch hätte beginnen können: im Feld der verschiedenen rituellen, spielerischen oder auch theatralen Formen, in denen Liturgie, Geistliches Spiel, Fastnachtsspiel und frühes Drama Schritt für Schritt eine Art performatives Kontinuum auszuformen scheinen, ohne dabei einem einheitlichen Typus verpflichtet zu sein.

Dass hinter diesem Befund auch die Schwierigkeit steckt, dass man den terminologischen Status des Ausdrucks, insbesondere angesichts seines erkennbar metaphorischen Gebrauchs durch Michel Foucault, bisweilen generell bezweifeln kann, sollte dabei durchaus festgehalten werden. Nur ist es gerade seine spezifische Vagheit, die den Ausdruck begrifflich in historischer Hinsicht so interessant macht.

C. Ackermann ( $\bowtie)$

Seminar für Deutsche Philologie, Universität Mannheim, Mannheim, Deutschland

E-Mail: christiane.ackermann@uni-mannheim.de

H. Bleumer

Seminar für Deutsche Philologie, Georg-August-Universität Göttingen, Göttingen, Deutschland

E-Mail: hbleume@gwdg.de 
Wenn ein Dispositiv jeweils das ist, was den Blick im Diskurs lenkt und Bilder und Begriffseinsichten hervorbringt, ohne dabei selbst sichtbar zu sein, wenn Dispositive im weitesten Sinne unsichtbare Blickkonzepte des Sichtbaren sind, dann müssten die Anfänge der Geschichte des Schauspiels für eine eigene Dispositiv-Diskussion interessant sein. Weil sich für die ästhetische Realisierung dessen, was die historische Forschung mit dem gezielt weiten Sammelbegriff des >Spiels< fasst, zum einen der antike, symbolische Rahmen des Theaters nicht mehr und seine neuzeitliche, performative Form noch nicht ansetzen lässt, weil von dieser ästhetischen Realisierung aus aber der lange, weitverzweigte Weg des Dramas in die Illusion beginnt, sollte sich gerade am Spiel und am frühen Drama der besondere Aufschlusswert der Dispositiv-Metapher zeigen lassen. Denn sie vermag darauf aufmerksam zu machen, dass in der Zeit vor dem institutionalisierten Theater und dem Blickregime der Guckkastenbühne die Funktion der Dispositive noch sprachlich, gestisch oder räumlich auf ganz unterschiedliche Weise realisiert, grade in dieser Variabilität aber beobachtbar wird.

Weil man sich zur wissenschaftlichen Beschreibung dieser Funktion nicht aus seiner eigenen medienhistorischen Vorprägung mit ihren Dispositiven herausphilosophieren kann, ist zugleich zur historischen Beschreibung dieser Funktion die Orientierung an der gezielt theatergeschichtlich ausgerichteten, modernen und postmodernen medientheoretischen Dispositivdiskussion unabdingbar, die sich insbesondere auf den frühen Vorschlag von Jean-Louis Baudry bezieht. In diesem Sinne wäre das dramatische Dispositiv - hier zunächst vereinfacht - als >apparative Anordnung < zu verstehen, dessen Grundstruktur aus der Konstellation von Publikum und Darstellenden sowie dem daraus resultierenden Raum und der simultan konfigurierten Perspektive entsteht. Diese Grundstruktur müsste sich dem Untersuchungsgegenstand angemessen historisieren lassen.

Der skizzierte, doppelte theoretische Zugriff wird - auf der Grundlage des vorbereitenden Exposés zum Heft - im einleitenden Beitrag Hartmut Bleumers näher erläutert. Das fragliche Doppelkonzept könnte demnach nicht zuletzt stärker dafür sensibilisieren, dass die Forschung nicht nur historische Dispositive zu beobachten vermag, sondern dazu auch selbst in historischen Dispositiven gefangen ist, und zwar nicht nur in solchen, die aus dem Fundus der dramatischen Formen stammen, sondern auch in den Mustern, die aus dem Haushalt narrativer Formen bekannt sind. Schon dies zeigen nämlich die Beiträge dieses Heftes sehr deutlich: Das wohlbekannte Narrativ einer Geschichte, die vom Spiel zum Drama und zum Theater führt, ist eine Wissenschaftsfiktion, die das historische Feld perspektivisch verzerrt und verkürzt. Setzt man stattdessen verschiedene dramatische Dispositive in diesem Feld an, so scheint sich eine Art Kaleidoskop der Phänomene zu ergeben, in dem diese sich von Fall zu Fall wechselseitig spiegeln und so, trotz permanenter Abweichungen, doch insgesamt aufklären.

Im Sinne dieser methodischen Pointe verstehen wir insbesondere die Beiträge von Elke Koch und Hans Rudolf Velten. So nimmt Koch ältere liturgische Praktiken vor der Zeit der Spielüberlieferung unter Problematisierung der dichotomischen Gegenüberstellung von >Ritual versus Theater $<$ sowie >Kult versus Kunst< in den Blick. In der historisch-kulturellen Relevanz liturgischer Praktiken erweist sich deren dispositive Wirksamkeit. Sie zeugen von einer dem telelogischen Entwicklungsnarra- 
tiv zuwiderlaufenden Interdependenz performativer Praktiken und volkssprachiger Spiele, wie Koch über ihren Begriffsvorschlag der >Rollentransparenz « verdeutlicht. Diese ermöglicht die Emergenz eines semantischen Mehrwerts, indem soziale Gemeinschaft und religiöses Wissen aufeinander hin transparent $\mathrm{zu}$ werden scheinen und solchermaßen einander Bedeutung zuweisen.

Demgegenüber setzt Velten an der materialen Seite der Performanz an. Er richtet seinen Fokus auf einfachste, gerade darin ästhetisch hochwirksame Hilfsmittel zur Markierung einer Spielfläche und damit auf die »Nullstufe der Bühne«. Daraus ergibt sich notwendig ein Plädoyer für die Berücksichtigung aller belegten Bühnenformen von 1300-1600 und damit einhergehend für eine Neubewertung vermeintlich dominierender Aufführungsprinzipen.

Den sich in der Diskussion andeutenden Perspektivenwechsel vom Theater als Dispositiv zu den Dispositiven des Theaters fordert explizit Christian Schmidt über den Dispositiv-Begriff und verdeutlich im Zusammenhang seines imaginationshistorischen Ansatzes, worin der theoretische Mehrwert des Dispositiv-Konzepts Michel Foucaults liegen könnte. Mit seinem Anschluss an das Andachtsmodell führt er das bisherige Forschungskonzept fort, indem er das Dispositiv der Andacht als ein Netz von Subjektivierungsstrategien versteht, in das auch das Geistliche Spiel und das frühe geistliche Drama involviert sind.

Der kollektiven Identitätsstiftung hingegen ist der kunsthistorische Beitrag von Anna Pawlak und Sophie Rüth gewidmet. Sie nehmen mit dem Beispiel Antwerpens die vormoderne Stadt als soziales Dispositiv ins Visier und erläutern das darin wirksame Zusammenspiel verschiedener kulturprägender Diskurse und Medien in ihrer Bedeutungs- und identitätsstiftenden Funktion anhand eines zentralen Mythos des städtischen Selbstbildes.

Fallbeispiele europäischer Festkultur bietet auch der Beitrag von Rebekka Nöcker, der einer klassisch-philologischen und medienhistorischen Methodik verpflichtet ist. Er konzentriert sich auf den Verschriftlichungsprozess von Spielen als Teil der Nürnberger Festkultur und untersucht die damit einhergehende neue narrative Qualität der sogenannten Nebentexte in Fastnachtspielen. Das Spektrum dieser Qualität zeigt der Beitrag kommentierend an zahlreichen Beispielen auf und exemplifiziert so die historische Tragfähigkeit des Dispositiv-Begriffs.

Den nahezu umgekehrten Fall, die Entstehung des auf Lektüre angelegten dramatischen Textes, der das Dispositiv des Theaters emergieren lässt, behandelt abschließend Christiane Ackermann in Fokussierung jener Zeit, in der sich das Theater zu institutionalisieren beginnt. Im Rekurs auf Baudrys Dispositiv-Konzept skizziert der Beitrag Elemente dramatischer Dispositive der Vormoderne und zeigt, dass sowohl das Theater als mediales Dispositiv des Dramas als auch das Drama als mediales Dispositiv des Theaters Wirkung entfalten kann.

Abschließend ist festzuhalten, dass die Beiträgerinnen und Beiträger auch unter den schwierigen Bedingungen des Corona-Lockdowns, die gerade vor dem Hintergrund der Sorgfaltspflichten philologischer Arbeit den Digitalisierungsoptimismus Lügen gestraft haben, ihren Publikationszusagen nachgekommen sind. Das vollständige und zeitnahe Zustandekommen des Heftes ist daher nicht zuletzt Ausdruck des persönlichen Engagements aller Mitwirkenden. Dafür haben wir an dieser Stelle ausdrücklich zu danken. 
Funding Open Access funding enabled and organized by Projekt DEAL.

Open Access Dieser Artikel wird unter der Creative Commons Namensnennung 4.0 International Lizenz veröffentlicht, welche die Nutzung, Vervielfältigung, Bearbeitung, Verbreitung und Wiedergabe in jeglichem Medium und Format erlaubt, sofern Sie den/die ursprünglichen Autor(en) und die Quelle ordnungsgemäß nennen, einen Link zur Creative Commons Lizenz beifügen und angeben, ob Änderungen vorgenommen wurden.

Die in diesem Artikel enthaltenen Bilder und sonstiges Drittmaterial unterliegen ebenfalls der genannten Creative Commons Lizenz, sofern sich aus der Abbildungslegende nichts anderes ergibt. Sofern das betreffende Material nicht unter der genannten Creative Commons Lizenz steht und die betreffende Handlung nicht nach gesetzlichen Vorschriften erlaubt ist, ist für die oben aufgeführten Weiterverwendungen des Materials die Einwilligung des jeweiligen Rechteinhabers einzuholen.

Weitere Details zur Lizenz entnehmen Sie bitte der Lizenzinformation auf http://creativecommons.org/ licenses/by/4.0/deed.de. 